\title{
A 6-12 GHz analogue lag-correlator for radio interferometry
}

\author{
C. M. Holler ${ }^{1,2}$, T. Kaneko ${ }^{1}$, M. E. Jones ${ }^{3}$, K. Grainge ${ }^{1}$, and P. Scott ${ }^{1}$ \\ 1 Cavendish Laboratory, Cambridge University, Cambridge CB3 0HE, UK \\ e-mail: tk229@mrao.cam.ac.uk \\ 2 Ludwigshohenweg 11, 83253 Rimsting, Germany \\ e-mail: holler@cantab.net \\ 3 Sub.-Dept. of Astrophysics, University of Oxford, Denys Wilkinson Building, Keble Road, Oxford OX1 7RH, UK
}

Received 5 December 2006 / Accepted 22 December 2006

\begin{abstract}
Aims. We describe a 6-12 GHz analogue correlator that has been developed for use in radio interferometers.

Methods. We use a lag-correlator technique to synthesis eight complex spectral channels. Two schemes were considered for sampling the cross-correlation function, using either real or complex correlations, and we developed prototypes for both of them. We opted for the "add and square" detection scheme using Schottky diodes over the more commonly used active multipliers because the stability of the device is less critical.

Results. We encountered an unexpected problem, in that there were errors in the lag spacings of up to ten percent of the unit spacing. To overcome this, we developed a calibration method using astronomical sources which corrects the effects of the non-uniform sampling as well as gain error and dispersion in the correlator.
\end{abstract}

Key words. instrumentation: interferometers - techniques: interferometric - radio continuum: general

\section{Introduction}

High brightness sensitivity is essential for many observations in radio astronomy, for example, observations of the cosmic microwave background $(\mathrm{CMB})$. Once the angular resolution required has been fixed by the size of the instrument in wavelengths, the brightness sensitivity is determined by two factors: the system temperature and the observing bandwidth. System temperatures have been reduced dramatically by the use of cryogenic HEMT amplifiers, to the point where the atmosphere rather than the amplifier may be the dominant noise source in the system, and thus little further improvement is possible for ground-based instruments. Increased continuum bandwidth is therefore an important route to increased sensitivity.

Radio interferometers with any significant bandwidth require the band to be broken up in to sub-bands, both because spectral resolution may be intrinsically required for the observation, and to overcome the effects of chromatic aberration. Digital correlators are commonly used since they provide the most convenient way of generating high spectral resolution and a large range of time delays. However, for very high brightness sensitivity continuum observations which demand maximum possible bandwidth with only modest spectral resolution, analogue correlators are competitive. Analogue correlators have been used in several recent interferometers dedicated to $\mathrm{CMB}$ observations (O'Sullivan et al. 1995; Lo et al. 2001; Padin et al. 2002; Leitch et al. 2002; Watson et al. 2003).

To achieve the low spectral resolution typically required (of order ten spectral channels, rather than the hundreds or thousands typical of digital correlators) there are two basic solutions. One is to split the broadband signal directly into smaller bands with filter banks or multiple downconverters (e.g. Padin et al. 2001). The other is to use a single broad intermediate frequency (IF) band and implement a Fourier transform spectrometer, or lag correlator. This forms the cross-correlation between antennas at multiple delays, and the outputs are Fourier transformed to estimate the cross-power frequency spectrum. In this paper, we describe the development of an analogue $6 \mathrm{GHz}$ bandwidth lag correlator with eight complex bands. The correlator is currently operational in the Arcminute Microkelvin Imager (AMI, see, for example Kneissl et al. 2001; Jones 2002; Kaneko \& the AMI Collaboration 2006), a new radio interferometer operating over the $12-18 \mathrm{GHz}$ band. AMI was specifically designed to carry out a survey for clusters of galaxies through the Sunyaev-Zel'dovich effect (Sunyaev \& Zel'dovich 1972). Broadband analogue correlators with similar concepts have been described by Harris \& Zmuidzinas (2001), Li et al. (2004), Roberts et al. (submitted). A feature that distinguishes our design from these correlators is the detectors. Rather than active multipliers, we chose the "add and square" detection scheme using zero-biased Schottky diodes. They are simpler to operate because no bias current is required and their stability is not as critical as it is for active devices. It is technically challenging to design IF components that span over more than one octave in frequency so we chose an IF band of 6-12 GHz. To reduce costs and complexity, we implemented the correlator using microstrip technology and each baseline was fabricated on a single substrate.

Section 2 of this paper discusses the basic relationships for a broadband lag correlator and discusses real vs. complex correlators and phase-switched multipliers. Section 3 describes the detector circuit in our design. Section 4 then describes the overall hardware implementation, and Sect. 5 the calibration scheme implemented to recover accurate spectra from the lag data in the presence of dispersion and lag spacing errors. Section 6 
describes issues related to the manufacturing and practical implementation of the correlator, with conclusions in Sect. 7.

\section{Correlator overview}

\subsection{Fourier transform correlator}

The response of an interferometer baseline with a single real correlator having a rectangular passband of width $\Delta v$ is given by

$R=R_{0} \frac{\sin \left[\pi \Delta v\left(\tau_{\mathrm{g}}-\tau_{\mathrm{i}}\right)\right]}{\pi \Delta \nu\left(\tau_{\mathrm{g}}-\tau_{\mathrm{i}}\right)} \cos \left[2 \pi\left(\nu_{\mathrm{LO}} \tau_{\mathrm{g}}-\nu_{\mathrm{IF}}\left(\tau_{\mathrm{g}}-\tau_{\mathrm{i}}\right)\right)+\phi_{\mathrm{LO}}\right]$

where $R_{0}$ is a normalising factor, $\tau_{\mathrm{g}}$ and $\tau_{\mathrm{i}}$ are geometric and instrument delays (i.e. the delays introduced at the radio frequency (RF) and IF respectively), $v_{\mathrm{LO}}$ is the local oscillator (LO) frequency, $\nu_{\mathrm{IF}}$ is the IF frequency and $\phi_{\mathrm{LO}}$ is the phase difference between the LO at each aerial. The cosine term describes the fringes that change with changing delay. The path compensator inserts an instrument delay $\tau_{\mathrm{i}}$ which (approximately) balances out the geometrical delay. The sinc term produces a fringe envelope, which maximises the response if the residual delay, $\left(\tau_{\mathrm{g}}-\tau_{\mathrm{i}}\right)$, is small. For simplicity, the phase difference in the LO at the two mixers, $\phi_{\mathrm{LO}}$, can be dropped as it is easily removed by calibration. Substituting for the LO frequency $v_{\mathrm{LO}}$ using the relationship $v_{\mathrm{LO}}-v_{\mathrm{IF}}=v_{\mathrm{RF}}$ for lower sideband reception,

$R=R_{0} \frac{\sin \left[\pi \Delta v\left(\tau_{\mathrm{g}}-\tau_{\mathrm{i}}\right)\right]}{\pi \Delta v\left(\tau_{\mathrm{g}}-\tau_{\mathrm{i}}\right)} \cos 2 \pi\left(\nu_{\mathrm{RF}} \tau_{\mathrm{g}}+v_{\mathrm{IF}} \tau_{\mathrm{i}}\right)$.

Chromatic aberration is a problem because of the extent of the observing field of view. While it is possible to introduce the correct instrumental delay to compensate for the geometrical delay at the centre of the field, a radio source a small angle $\Delta \theta$ away from the field centre will have an additional geometric delay $D \Delta \theta / c$, where $D$ is the baseline length projected on to the plane perpendicular to the source direction. If it is required to image with minimal loss of sensitivity to the edge of the primary beam of an interferometer with an antenna diameter $d$

$\pi \frac{\Delta v / \nu_{\mathrm{RF}}}{d / D} \ll 1$

(see e.g. Thompson et al. 2001). To satisfy this requirement for an instrument with centre frequency $v_{\mathrm{RF}}=15 \mathrm{GHz}$ and a maximum ratio of baseline to dish diameter $D / d \simeq 10$ we chose to split the full $6 \mathrm{GHz}$ bandwidth into eight sub-bands of $0.75 \mathrm{GHz}$ each.

The basic layout of the Lag or Fourier transform correlator is illustrated in Fig. 1a. The signals $A(t)$ and $B(t)$ from the two antennas of a baseline are correlated at discrete time delays $\tau_{\mathrm{i}}$ with step size $\delta \tau$. The set of correlated signals form the crosscorrelation function:

$R\left(\tau_{\mathrm{i}}\right)=\frac{1}{T} \int_{0}^{T} A(t) B\left(t+\tau_{\mathrm{i}}\right) \mathrm{d} t$,

where $T$ is the integration time. This type of correlator measures the temporal coherence function of a signal, where the coherence function is an indirect measure of the signal's frequency spectrum. The cross-power spectrum (hereafter abbreviated to the spectrum) can be recovered by applying the discrete Fourier transform (DFT) to the cross-correlation function;

$S\left(v_{k}\right)=\sum_{\tau_{\mathrm{i}}} R\left(\tau_{\mathrm{i}}\right) \mathrm{e}^{-2 \pi j v_{k} \tau_{\mathrm{i}}}$

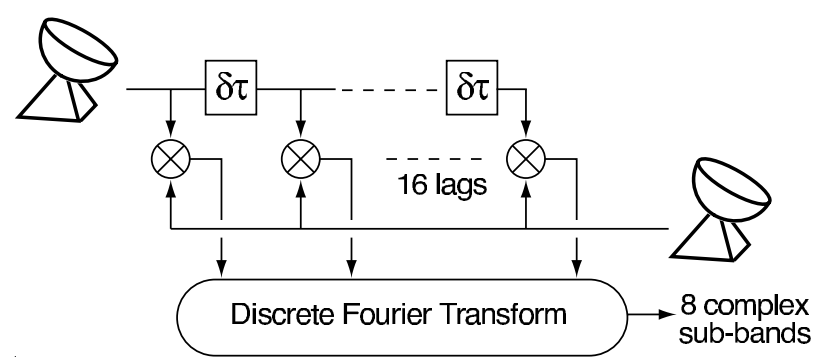

(a) Cross-correlation function

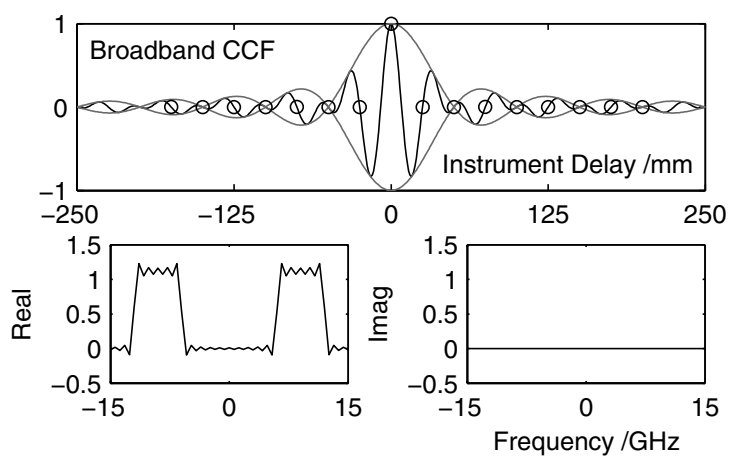

(b) Real Correlator

Fig. 1. a) The lag correlator cross-correlates the signals from a pair of antennas at discrete delay steps (denoted by $\delta \tau$ ). Applying a discrete Fourier transform to the set of lag data gives the complex power spectrum. The real correlator samples at 16 lags. b) The cross-correlation function and the spectrum. The gray envelope is the bandwidth pattern and the open circles are the positions of the lags. For a source at the centre of the field of view and no geometric delay, the imaginary component of the spectrum is zero. In general, the real correlator has complex spectra at positive and negative frequencies.

Figure 2 illustrates typical cross-correlation functions of a flatspectrum point source at the telescope's phase centre for a narrowband and broadband system with rectangular passband. The function is given by Eq. (2) with $\tau_{\mathrm{g}}=0$ and $\tau_{\mathrm{i}}$ as the variable. The cosine term has a period determined by the centre IF frequency $v_{\mathrm{IF}}$ and the sinc envelope is defined by the bandwidth $\Delta v$. As the source moves across the sky, the cosine pattern drifts in instrument delay space. The envelope encodes the spectral information and its Fourier transform gives the spectrum of the observed signal. The desired information is contained in the envelope and it must be sampled at Nyquist sampling step, $\delta \tau_{\mathrm{N}}=1 /(2 \Delta v)$.

A point source at the edge of the field of view will introduce an additional geometrical time delay $\Delta \tau_{\mathrm{g}}$ into one arm of the correlator. The additional delay will offset the cross-correlation function away from the centre lag. We define a narrowband correlator to be one where this delay $\Delta \tau_{\mathrm{g}}$ is shorter than the Nyquist sampling step $\delta \tau_{\mathrm{N}}$. In order to avoid chromatic aberration, no additional lags are necessary. However in a broadband system, where $\Delta \tau_{\mathrm{g}}$ is greater than $\delta \tau_{\mathrm{N}}$, the offset cannot be neglected. For our design parameters, $\Delta \tau_{\mathrm{g}}$ can be up to $\pm 6 \delta \tau_{\mathrm{N}}$ so the crosscorrelation function has to be sampled over a wide interval. We sample 16 lags at $-8 \delta \tau_{N},-7 \delta \tau_{N}, \ldots+7 \delta \tau_{N}$. After applying the DFT, this splits the passband into eight complex sub-bands of $0.75 \mathrm{GHz}$ each. Most of the signal power is concentrated in the centre of the cross-correlation function's envelope. Its position in instrument delay space depends on the position of the source in the field of view and must be within the range of the sampled lags. Figure 3 shows simulations of the amplitude response for each output channel of a 16-lag correlator as a function of 


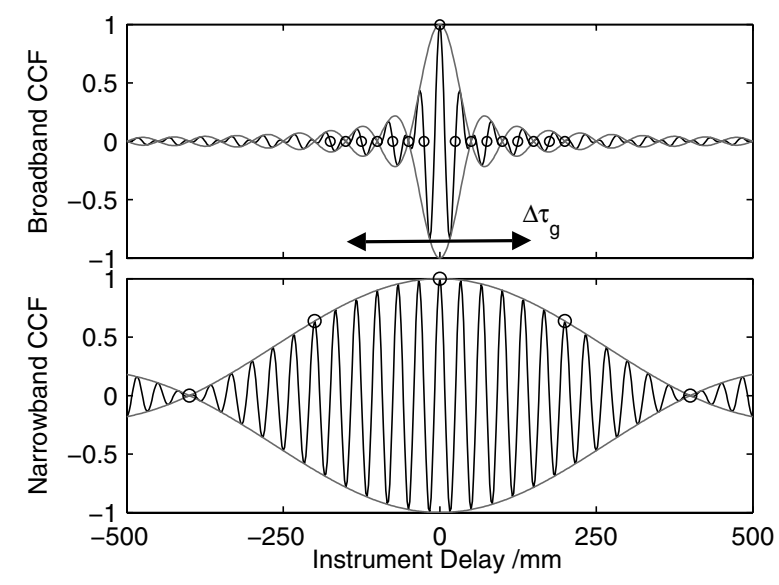

Fig. 2. The cross-correlation functions for a broadband $\left(v_{\mathrm{IF}}=9 \mathrm{GHz}\right.$, $\Delta v=6 \mathrm{GHz})$ and a narrowband system $(\Delta v=0.75 \mathrm{GHz})$. The horizontal scales are instrument delay in electrical length. The gray envelopes are the bandwidth patterns and the open circles are the lag positions. A source at the edge of the field of view will introduce an additional geometrical time delay $\Delta \tau_{\mathrm{g}}$ and offset the cross-correlation function over the range of the arrow.

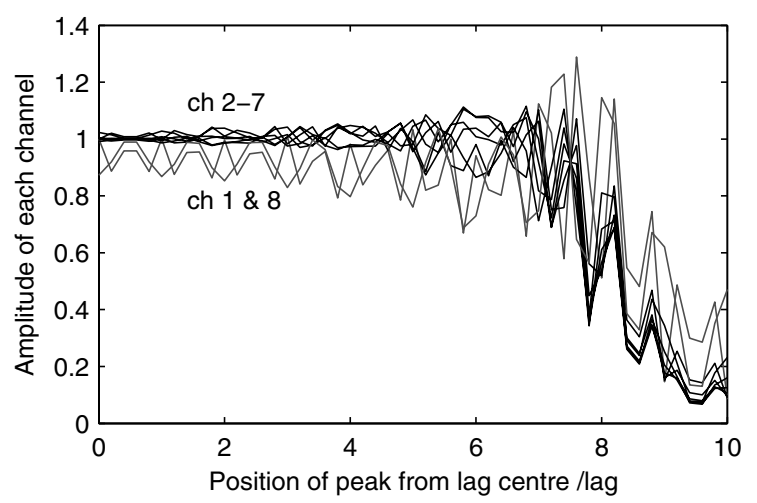

Fig. 3. The amplitude response of each frequency channel of the correlator to the broadband cross-correlation functions (Fig. 2) positioned away from the lag centre. This is equivalent to point sources away from the pointing centre. The sensitivity of the correlator is fairly constant over the field of view, corresponding to a maximum of \pm 6 lags. The structures and the reduced sensitivity in the edge sub-bands 1 and 8 will be discussed in Sect. 5.2.

the centre position of the cross-correlation function. By using 16 lags for the correlator, its sensitivity is not significantly reduced for sources within the telescope's field of view.

\subsection{Signal recovery}

A narrowband signal of centre frequency $v$ can be sampled by a simple single-lag correlator. The signal received at each antenna is a real quantity but it is convenient to express it in complex notation. The signals at the two antennas are the real components of

$A(t)=a \mathrm{e}^{j(2 \pi v t+\phi)} \quad$ and $\quad B(t)=b \mathrm{e}^{j 2 \pi v t}$,

where $\phi$ is the signal phase which depends on the position of the source and geometry of the interferometer. After correlating these signals, the output of the correlator is

$\operatorname{Re}\left[A(t) B^{*}(t)\right]=\operatorname{Re}\left[a b \mathrm{e}^{j \phi}\right]=a b \cos \phi$.
We take the complex conjugate of $B(t)$ because we are interested in the product of the real parts. The quantities we want to measure are the amplitude $a b$ and the phase $\phi$. To measure them both simultaneously, we would need two measurements. We could make the second measurement by inserting a $90^{\circ}$ phase shift in one arm so that

$A^{\prime}(t)=a \mathrm{e}^{j(2 \pi v t+\phi+\pi / 2)}$.

The second measurement will be

$\operatorname{Re}\left[A^{\prime}(t) B^{*}(t)\right]=\operatorname{Re}\left[a b \mathrm{e}^{j(\phi+\pi / 2)}\right]=a b \sin \phi$.

From these in-phase (Eq. (3)) and quadrature (Eq. (4)) components, we can simultaneously measure the amplitude and phase of the signal. As mentioned earlier, the Fourier transformed output of the lag correlator is a complex spectrum. This is a way of expressing the amplitude and phase of each sub-band. Similarly, we could express the amplitude and phase measured by the single-lag correlator as a complex value. We stress that the crosscorrelation function is a real quantity and the complex notation is for mathematical convenience. To reflect this notation, we will refer to the in-phase and quadrature components as real and imaginary.

In a multiple-lag correlator, however, we have two options. We could either sample just the in-phase components of the cross-correlation function (real correlator - see Fig. 1a) or both the in-phase and quadrature components (complex correlator - see Fig. 4a). The choice will affect the form of the spectrum $S\left(v_{k}\right)$. But the information we recover is equivalent.

\subsubsection{Real correlator}

Here, $2 n$ real components of the cross-correlation function are sampled at Nyquist rate with step size $\delta \tau_{\mathrm{N}}$; an electrical length of $25 \mathrm{~mm}$ or an equivalent sampling frequency of $12 \mathrm{GHz}$ for a $6-\mathrm{GHz}$ bandwidth signal. The cross-correlation function is sampled from $-n \cdot \delta \tau_{\mathrm{N}}$ to $+(n-1) \cdot \delta \tau_{\mathrm{N}}$, where $n$ is the number of complex sub-bands ${ }^{1}$. The symmetry between the positive and negative halves of the spectrum means that half the information in the spectrum is redundant. 16 independent measurements of the cross-correlation function give 8 independent complex subbands (see Fig. 1b).

\subsubsection{Complex correlator}

The second method samples both the real and imaginary components of the cross correlation function but at only half-Nyquist rate $(6 \mathrm{GHz}$ sampling frequency, or an equivalent electrical length of $50 \mathrm{~mm}$ ). We express the measurements as a set of $n$ complex numbers. The step size is $2 \delta \tau_{\mathrm{N}}$ and samples span from $-n \cdot \delta \tau_{\mathrm{N}}$ to $+(n-2) \cdot \delta \tau_{\mathrm{N}}$. The resulting spectrum contains only positive frequencies (Fig. 4b). To measure the imaginary component, one arm of input signal has a broadband $90^{\circ}$ phase shifter ${ }^{2}$. 8 complex measurements will give 8 complex sub-bands. The single-sided spectrum explains why half-Nyquist sampling is

${ }^{1}$ Generally, the spectrum will be complex in both positive and negative frequencies. These two components are Hermitian; $S(v)=S^{*}(-v)$, where the asterisk denotes the complex conjugate. The relationship could be observed from the symmetry in the spectrum.

2 The phase shift produces a Hilbert transform of the original signal; for a single-sided spectrum, the real part of the cross-correlation function is the Hilbert transform of its imaginary part. 


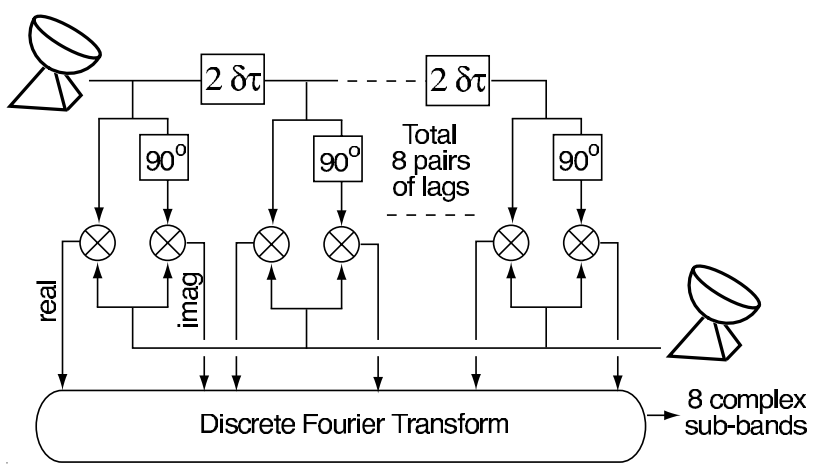

(a) Complex Correlator

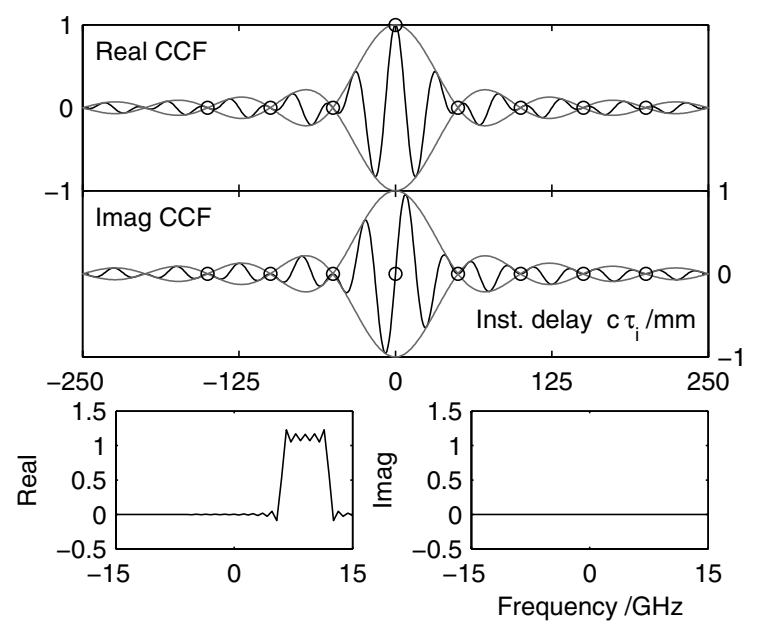

(b) Cross-correlation function

Fig. 4. a) The complex correlator samples at only eight lags but with two measurements per lag and twice the delay steps. Using the $90^{\circ}$ phase shifters, it samples the in-phase and quadrature components of the cross-correlation function at each lag. b) The cross-correlation function and the spectrum. In general, the spectrum recovered by the complex correlator is single-sided.

sufficient. It can also be understood in terms of information content: the information in 8 complex measurements is the same is 16 real measurements.

To explore possible practical differences between the two methods, we built prototypes for both schemes.

\subsection{Cross-correlation and phase-switching}

There are several ways of correlating two analogue signals. In a single baseline interferometer, two antennas measure random noise-like signal voltages $A(t)$ and $B(t)$. We will represent these by random variables $\boldsymbol{a}$ and $\boldsymbol{b}$. Active multipliers will directly give the product $\boldsymbol{a} \boldsymbol{b}$. Active components have higher sensitivity but their stability is often more critical. The relative merits of active multipliers are discussed in Harris (2003).

We use a very simple concept, described by Ryle (1952) as "add and square" detection. The two signals from the antennas are summed to give $\boldsymbol{a}+\boldsymbol{b}$. The summed signal is then passed through a non-linear device, in our case a diode, with an output of the form

$(a+b)^{2}=a^{2}+2 a b+b^{2}$.

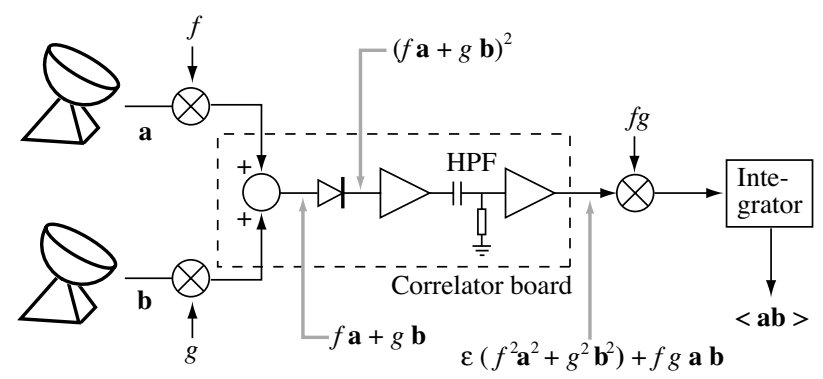

Fig. 5. The "add and square" analogue correlator including phase switching. The signal from the detector is amplified and most of the total power terms are removed by the highpass filter (HPF). The HPF attenuates the direct current (DC) signal by a factor $\epsilon$. Any remaining total power and DC offsets are removed by phase-switch demodulation. $\langle\cdot\rangle$ is the expectation.

The total power terms of each antenna $\boldsymbol{a}^{2}$ and $\boldsymbol{b}^{2}$ dominate the output, and the cross-correlation term $\boldsymbol{a b}$ needs to be extracted before recording the data. We use phase-switching to remove the total power terms, which also reduces slowly varying offsets and cross-talk in the system. The detection scheme is illustrated in Fig. 5. The two signals are modulated by Walsh functions $f$ and $g$. These are periodic bi-valued functions which have the property that all the functions in a given family are orthogonal to each other, and to their product with any other Walsh function. Phase shifting the signal by $180^{\circ}$ applies the modulation. The output from the correlator will be

$(f \boldsymbol{a}+g \boldsymbol{b})^{2}=f^{2} \boldsymbol{a}^{2}+2 f g \boldsymbol{a} \boldsymbol{b}+g^{2} \boldsymbol{b}^{2}$.

The products of Walsh functions with themselves, $f^{2}$ and $g^{2}$, are constants. The product of two different Walsh functions, $f g$, is another Walsh function. To extract the cross-term, the output is demodulated by $\mathrm{fg}$ and integrated over the repeat period $T_{\mathrm{w}}$ of the Walsh function:

$\frac{1}{T_{\mathrm{w}}} \int_{0}^{T_{\mathrm{w}}} f g\left(f^{2} \boldsymbol{a}^{2}+2 f g \boldsymbol{a} \boldsymbol{b}+g^{2} \boldsymbol{b}^{2}\right) \mathrm{d} t \propto \frac{1}{T_{\mathrm{w}}} \int_{0}^{T_{\mathrm{w}}} \boldsymbol{a} \boldsymbol{b} \mathrm{d} t$.

Phase-switching also removes any slowly varying drift on timescales longer than the repeat period. It also suppresses crosstalk because signals from other antennas will be modulated with an orthogonal Walsh function.

To achieve the full signal-to-noise ratio (SNR) in such a correlator, it is also necessary to form the "subtract and square" product $(\boldsymbol{a}-\boldsymbol{b})^{2}$. This can be seen by considering that each voltage being correlated consists of a signal term which is correlated between the two antennas and noise term that is uncorrelated. Since the noise terms are uncorrelated, two orthogonal signal combinations can be made from them with uncorrelated noises. The noise on the sum signal, $\boldsymbol{a}+\boldsymbol{b}$, is thus uncorrelated with the noise on the difference signal, $\boldsymbol{a}-\boldsymbol{b}$. Each can then be squared to form estimates of the product $\boldsymbol{a} \boldsymbol{b}$ with independent noises, and these can be added to form the full signal-to-noise product. A full plus-minus system is thus equivalent to a single real multiplication, and two such multipliers $90^{\circ}$ apart would make a single complex correlator.

A second correlator is thus equipped with a $180^{\circ}$ phase shift in one arm. The output from this second correlator will be

$(f \boldsymbol{a}-g \boldsymbol{b})^{2}=f^{2} \boldsymbol{a}-2 f g \boldsymbol{a} \boldsymbol{b}+g^{2} \boldsymbol{b}$.

In principle the plus and minus correlator outputs can be differenced in hardware and the signal then demodulated and read 


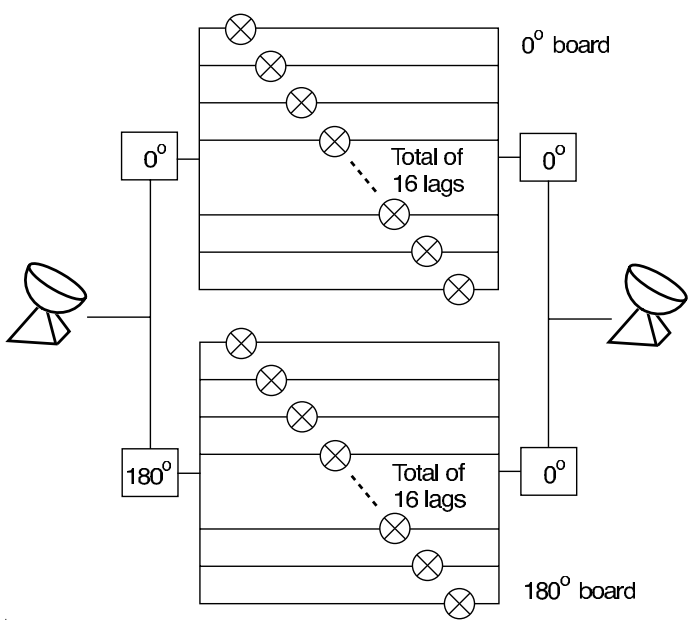

(a) Real correlator schematic layout

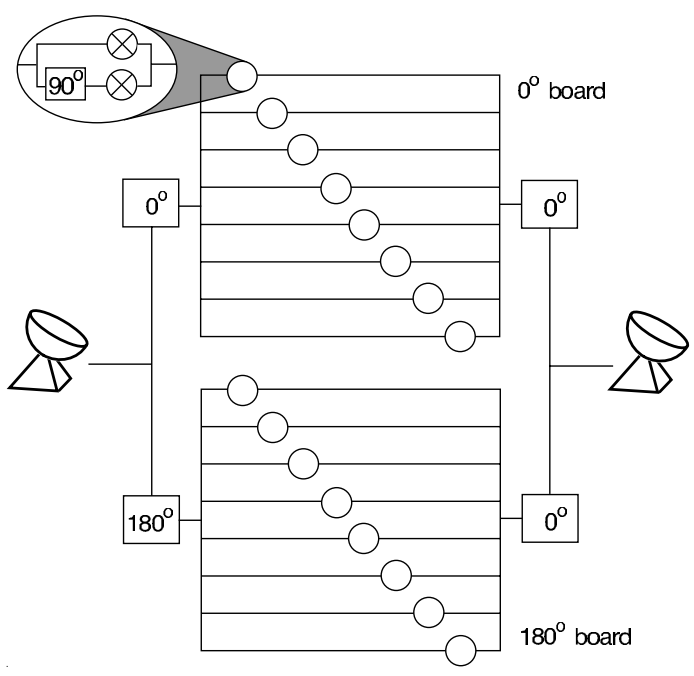

(b) Complex correlator schematic layout

Fig. 6. The full schematic of the real a) and the complex b) correlators for a single baseline. The real correlator samples at 16 lags in steps of $\delta \tau_{\mathrm{N}}$, while the complex correlator samples at 8 lags in steps of $2 \delta \tau_{\mathrm{N}}$. At each lag of the complex correlator, the signal is split and one arm is phaseshifted by $90^{\circ}$. In both cases, there is a $180^{\circ}$ board with one arm phase-shifted by $180^{\circ} .0^{\circ}$ phase shifters of similar slotline design are used in corresponding positions to match the response with that of the $180^{\circ}$ phase shifters.

out. This cuts down the necessary readout electronics by half. However, our final system demodulates and reads out the outputs from each correlator separately. This enables individual errors in the correlator lag positions to be corrected before the signals are combined. (see Sect. 4.2).

The full schematic drawings of the real and complex correlators are shown in Figs. 6a and b. The components for these correlators include broadband $(6-12 \mathrm{GHz})$ signal splitters, slotline $0^{\circ}$ and $180^{\circ}$ phase shifters, $90^{\circ}$ microstrip phase shifters for the complex correlator and the detector circuits, including the diodes. Our designs of the splitter and the phase shifters have been described in Holler \& Jones (submitted).

\section{Detector}

The detector circuit is the core of the correlator. We use a diode power detector circuit (Fig. 7) comprising a Schottky diode operated in the square law region. For $V_{\text {in }} \ll V_{\mathrm{T}}$, where $V_{\mathrm{T}}=k_{\mathrm{B}} T_{0} / e \simeq 26 \mathrm{mV}$ is the thermal voltage at room temperature $T_{0}$, the output voltage is proportional to input power (Hewlett-Packard AN 986).

For very small input signals a bias current could be used to increase the output. However, the output would be very sensitive to changes in this current with, for example, temperature, so we chose to use zero-biased diodes. The disadvantage is that they require a relatively high input power in the region of $-13 \mathrm{dBm}$ or $0.05 \mathrm{~mW}$. But they are very stable, as long as they are kept at a constant temperature.

Tradeoffs had to be made between the most important performance measures of the detector. These include sensitivity over the full band, stability, the video bandwidth (the lowpass-filtered bandwidth after the detector), avoiding it behaving like a peak detector and frequency matching.

The sensitivity largely depends on the value of the load resistor $R_{\mathrm{L}}$. A high value gives a higher output voltage for the same input power, but also reduces the video bandwidth for the signal behind the diode. Phase-switch modulation introduces sharp transitions in the signal. Slews over the transition will degrade

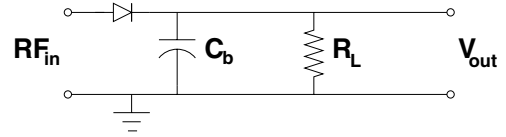

Fig. 7. The power detector circuit. The forward (direct) current through the diode produces a voltage across the load resistor $R_{\mathrm{L}}$. The bypass capacitor $C_{\mathrm{b}}$ looks like a short for the RF signal.

the orthogonality between the signals. For phase-switch signals with a maximum frequency of $1 \mathrm{kHz}$, we required a minimum video bandwidth of about $1 \mathrm{MHz}$. The bypass capacitor also has a large influence on the video bandwidth, but its value should not be too low since it acts as a ground for the RF.

Under certain circumstances, the detector could operate as a peak detector, where a large voltage peak charges up the bypass capacitor $C_{\mathrm{b}}$ and no more current can flow through the diode. This can be avoided if the detector is operated in the small signal regime where the output voltage is comparable to the thermal voltage $V_{\mathrm{T}}$. In this regime, the detector circuit will act as an integrator. Additionally, when operated in that regime, the detector's output voltage is proportional to the input power.

After testing several circuits the diode MSS20-146 from Metelics was chosen together with $R_{\mathrm{L}}=10 \mathrm{k} \Omega$ and $C_{\mathrm{b}}=10 \mathrm{pF}$. The results can be seen in Fig. 8. The passband is relatively flat and rather upwards sloping, which is advantageous, since most other microwave components will add a slope in the opposite direction. The features in the passband are very similar for all tested types of diodes, which means they can be attributed to the matching circuit rather than the diode. A plot of the diode response versus input power shows a square law region up to about $30 \mathrm{mV} \simeq V_{\mathrm{T}}$ and is consistent with the simulated model. The measured video bandwidth was in the range of $800-1100 \mathrm{kHz}$.

Matching the diode to the $50 \Omega$ microstrip circuit was an important task, both to maximise power transfer to the detector and to avoid setting up standing waves in the correlator signal distribution circuit. The diode includes reactive components so the impedance is complex as well as depending on input frequency. The minimum absolute value of the impedance for our 


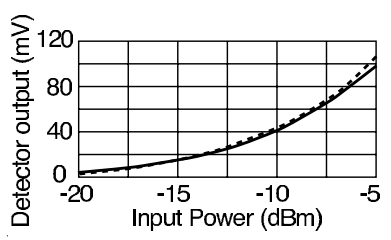

(a) Detector response

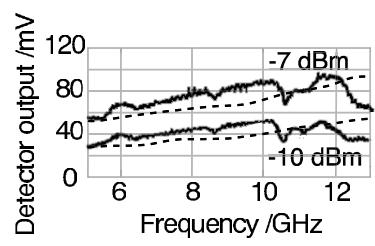

(b) Detector Passband
Fig. 8. a) The detector response against power for a $9 \mathrm{GHz}$ signal. The square law region extends to about $-11 \mathrm{dBm}$ input power. b) The passband of the detector (including the matching circuit) from 5 to $13 \mathrm{GHz}$. In both plots, the solid lines are the measurements and the dashed lines are simulations.
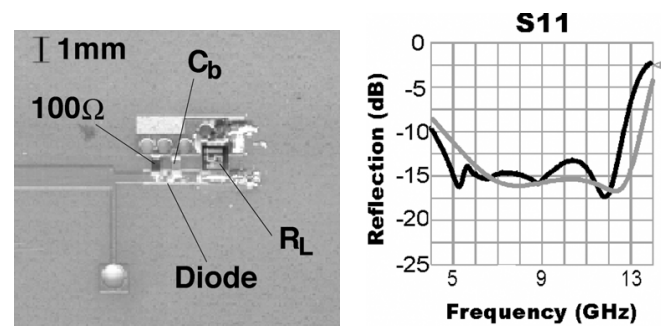

Fig. 9. A photograph of the final detector circuit (left) and the result of the matching (right). The plots show the measured input reflection (black curve) together with a simulation (gray curve).

frequency band can be calculated to about $100 \Omega$. To keep reflections and signal scatterings between channels to a minimum, we required $S_{11}$ to be low across the whole band. We used two element matching with a series microstrip line and a shunt line. In addition, the high impedance of the diode is reduced through a $100 \Omega$ parallel lumped resistor at the cost of losing sensitivity. The resistor will bypass part of the signal away from the diode which will not be detected. The characteristics of the final detector circuit are shown in Fig. 9.

\section{Correlator hardware}

\subsection{Design}

In the final design, $0^{\circ}$ and $180^{\circ}$ correlations for a single baseline (see Fig. 6) were implemented on two separate boards. Figures $10 \mathrm{a}$ and $\mathrm{b}$ show the physical layout of the $0^{\circ}$ real and $180^{\circ}$ complex boards. The designs show all the components; splitters, phase shifters and the detector circuits. To combine the signals before the detectors, we used Wilkinson dividers in reverse. Each lag has different delay lengths. The layouts were designed to avoid right angle bends and to keep components at a distance to try to minimise possible coupling. The layout of the complex correlator was very similar to the real correlator. The most significant differences are the $90^{\circ}$ phase shifters and different delay lengths.

\subsection{RF measurements}

The transmission characteristics of the correlator through to the input to the diode detector have been plotted in Fig. 11. The signal has cascaded through the $180^{\circ}$ slotline phase shifter, five signal splitters and one signal combiner (and one arm of the $90^{\circ}$ phase shifter in the case of the complex board). A lossless system would show a flat response at about $-15 \mathrm{dBm}$. But microstrip introduces loss, which is proportional to frequency and causes the slope across the band. The slope can be corrected for

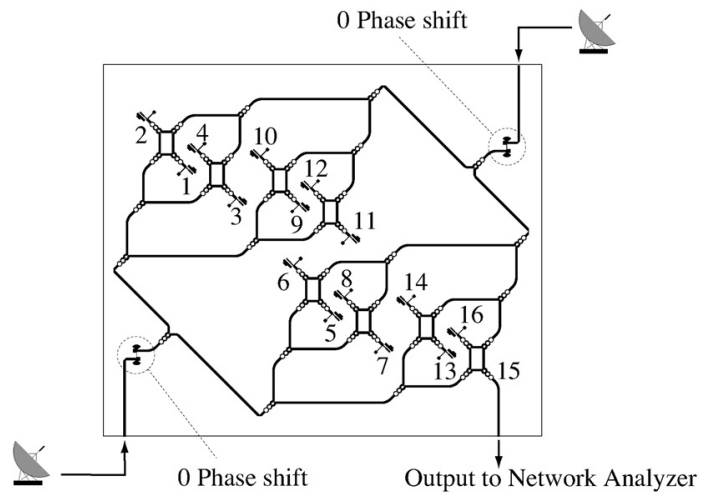

(a) Prototype $0^{\circ}$ Real Correlator

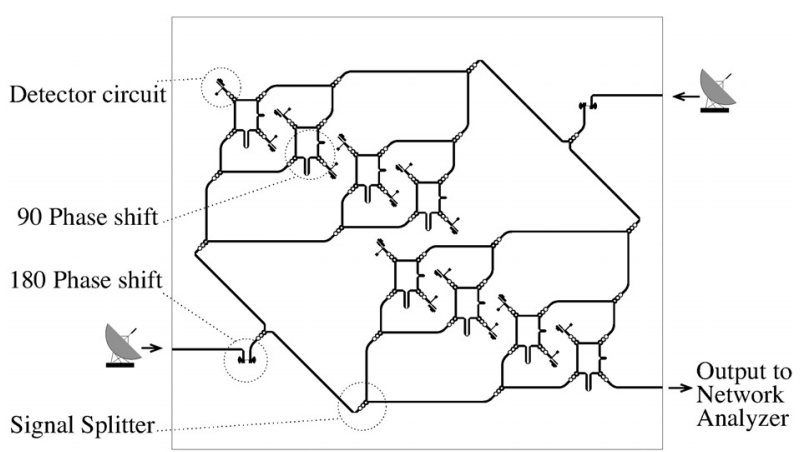

(b) Prototype $180^{\circ}$ Complex Correlator

Fig. 10. The physical layout of the $0^{\circ}$ real correlator board a) and the $180^{\circ}$ complex correlator board b). The corresponding $180^{\circ}$ real and $0^{\circ}$ complex correlator boards are not shown. For these prototype versions, one detector was swapped for a $50 \Omega$ output to enable measurements on the network analyser. The numbers on the real correlator correspond to the lag orders.
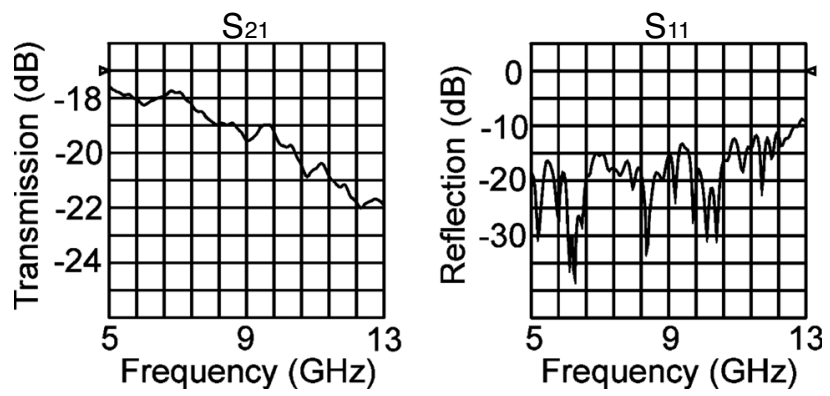

Fig. 11. Left: signal transmission through the correlator lags. Right: correlator input reflection. The results for the Complex and Real boards are very similar.

by inserting equalisers before the correlator input and is therefore of small concern to us. Large ripples across the band would be a problem, since they reduce the signal-to-noise performance of the correlator.

The performance of the phase shifters inside the correlator is shown in Fig. 12. The $180^{\circ}$ slotline phase shifter shows an exceptional behaviour with a maximum error of $\pm 6^{\circ}$ over the band of $5-13 \mathrm{GHz}$. The $90^{\circ}$ microstrip phase shifter has a maximum error of about $\pm 15^{\circ}$.

The frequency response, including all microstrip components, the detector circuit and the video band buffers of the prototype system is shown in Fig. 13a. There are some variations 


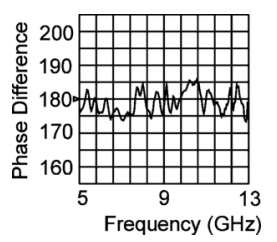

(a) $180^{\circ}$ shifter
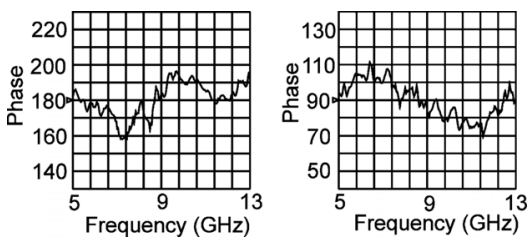

(b) $90^{\circ}$ Phase shifter
Fig. 12. a) Performance of the $180^{\circ}$ phase shifter in the system. For the performance of the $90^{\circ}$ phase shifter $\mathbf{b}$ ), the difference between these two curves has to be taken.

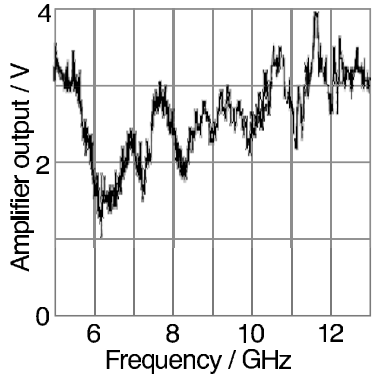

(a) Passband

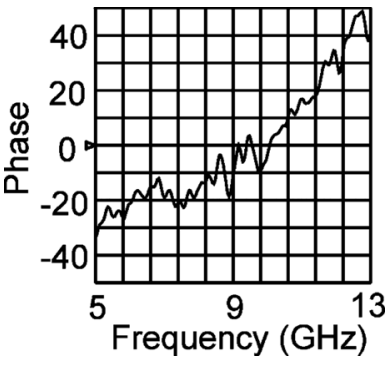

(b) Dispersion
Fig. 13. a) Typical passband of a full lag including all RF components plus the detector circuit and the two first stages of amplification. This shows a dip at $6 \mathrm{GHz}$ compared to the response of the detector in Fig. 8b. b) Dispersion in the outer most lags, which have the largest differential path length. The slope across the band is $\pm 25^{\circ}$.

across the band but it is relatively flat with a gentle slope. The rising passband is similar to the detector's passband (Fig. 8b) but there is a noticeable dip at $6 \mathrm{GHz}$. The structures in the passbands of the other lags differ slightly and the average signal levels vary by a factor of 2 to 3 . Therefore calibration of the correlator will be necessary.

Besides being lossy, particularly at higher frequencies, microstrip also exhibits dispersion. For our substrate with $\epsilon_{r}=9.7$ a physical length of one wavelength $(1.0 \lambda)$ at $6 \mathrm{GHz}$ is $2.065 \lambda$ at $12 \mathrm{GHz}$. However, this will have less influence on the correlator than one might expect. The two opposing signals in the correlator travel through similar length of microstrip line so dispersion is compensated. It is only the differential length of stripline that produces dispersion and the worst affected are the two outer lags. Figure $13 \mathrm{~b}$ shows a measurement for one of these lags. It shows a phase slope of $\pm 25^{\circ}$ across the band of 6-12 GHz, as expected from the distance to the zero lag. This implies that the electrical position of the point of correlation is a function of frequency. This effect will be compensated by the calibration process that will be discussed in Sect. 5.1.

The performances of the real and complex correlators were comparable. The ability to directly measure the amplitude and phase at each lag in the complex correlator is attractive for some commissioning tests. But calibrating for the small errors in the $90^{\circ}$ phase shifters over the passband adds an extra layer of complexity. From a practical view point, the more compact format of the real correlator fitted better into standard $6 \mathrm{U}$ racks. For these reasons, we chose the real correlators for the final system.

One set of measurements did not turn out as expected. The physical distance between the lags varied much more than anticipated. As a consequence, the cross-correlation function is not uniformly sampled and a standard DFT cannot be used to recover the true spectrum. Instead, a means of calibrating the correlator had to be found. The exact positions of the correlations could be measured and this information could be used to estimate the true spectrum. The lag position errors on the prototype board were apparently random with values of 5-10\% of the ideal lag spacing. However, in the final production series, the lag errors were found to be smaller than in the prototype boards. Nevertheless, they will cause some reduction in the SNR. Such lag errors have also been found in other analogue broadband correlators (Harris \& Zmuidzinas 2001; Roberts et al. submitted).

The causes of the lag errors are unclear. The lag errors are not consistent between boards so a design error is ruled out. By replacing lumped components, we determined that the lag spacings are insensitive to the exact positions of the components. Inspection of the boards showed no problems with the printing or production of the board. A solder mask layer is printed to protect the lines and restrict solder flowing along the tracks. A variation in this layer could influence the effective dielectric constant. We manufactured a correlator board without a solder mask but the lag errors persisted. Before the thin gold layer can be plated on the tracks, the surface of the copper is roughened. It is conceivable that the roughened surface could increase the path length at high frequencies because most of the current flows along the surfaces of the lines. To test this, we also manufactured a correlator board with no gold coating. Reliable measurements for this board could not be made because of poor contact to the connector.

We found that groups of lags had similar lag errors to each other. This indicated that these groups may have been affected by a long arm of the delay line shared by the whole group. Variations in the relative permittivity of the dielectric $\epsilon_{r}$ could be a cause but the errors in neighbouring lags are too large to be explained by this. Variations in $\epsilon_{r}$ of \pm 0.5 would be necessary to explain the average errors and only if the dielectric variations exactly followed the geometry of the board. This level of variation in $\epsilon_{r}$ is much higher than the manufacturing tolerance. Whatever the cause may be, the lag spacings are stable over time. This enables us to correct for the lag errors using a calibration scheme we have developed for the broadband correlators.

\section{Calibration}

\subsection{Method}

The eight complex frequency channels can be recovered from the 16 lag data by taking a direct Fourier transform (DFT). The DFT kernel can be conveniently expressed with the 16 by 16 DFT matrix

$\mathbf{F}=\left[\begin{array}{ccccc}W^{0} & W^{0} & W^{0} & \ldots & W^{0} \\ W^{0} & W^{1} & W^{2} & \ldots & W^{N-1} \\ W^{0} & W^{2} & W^{4} & \ldots & W^{2(N-1)} \\ \vdots & \vdots & \vdots & & \vdots \\ W^{0} & W^{N-1} & W^{2(N-1)} & \ldots & W^{(N-1)(N-1)}\end{array}\right]$

where $W^{k}=\exp (-2 \pi j k / N)$ and $N=16$. The input data are the 16 voltages measured at the lags, written as a column vector $\boldsymbol{v}_{0}$. The recovered spectrum is given by

$s_{0}=\mathbf{F} \boldsymbol{v}_{0}$.

Only the first eight of the elements in $s_{0}$ are used because the remaining eight are related to the first eight elements (see Sect. 2.2). This opens up a way for speeding up the DFT by adopting an 8 by 16 matrix. To keep things simple, we proceed with the full 16 by 16 matrix. 
DFT usually assumes uniform sampling but Sect. 4.2 showed that the sampling may be irregular by up to $10 \%$ because of errors in the lag spacing. In the absence of noise, the true spectrum can be recovered from non-uniformly sampled data - see, for example Bagchi \& Mitra (1998). We have developed a method that is particularly well suited for interferometers and uses an astronomical source for calibration. It can be shown that the degree of correlated noise between the lags is minimised when Nyquistsampled. In a correlator with lag errors, the noise becomes more correlated. The overall effect of non-uniformly sampled data is to degrade the SNR when there is noise - see, for example Bracewell (1999). The sampling errors are calibrated by defining a recovery matrix $\mathbf{R}$ that will return the true spectrum $\boldsymbol{s}_{0}$, given the non-uniformly sampled lag data $v$;

$\mathbf{F R} v=s_{0}$.

$\mathbf{R}$ can be thought of as a transformation that interpolates the non-uniformly sampled data $\boldsymbol{v}$ to the uniformly sampled data $\boldsymbol{v}_{0}$ that would have been measured had there been no lag errors. $\mathbf{R}$ also corrects for variations in the gain of individual detectors and dispersion in the microstrip delay lines. This is similar to the scheme used for the WASP2 lag correlation spectrometer (Harris \& Zmuidzinas 2001), except that WASP2 was calibrated using a CW signal to determine the recovery matrix $\mathbf{R}$. Because it is a laborious task to calibrate each correlator individually, we use an unresolved astronomical source to calibrate all correlators simultaneously in situ. In an ideal correlator, the cross-correlation function is bound by a sinc envelope. When Nyquist-sampled, the central peak of the sinc envelope lies on a lag and the nulls of the envelopes coincide with the other lags (when $\tau_{\mathrm{g}}=\tau_{\mathrm{i}}$ ). To form the 16 by 16 calibration matrix, the peak is aligned to the first lag. This will give a set of measurements like $\boldsymbol{v}_{1}=(1,0 \ldots 0)^{\mathrm{T}}$, where $\mathrm{T}$ is the transpose. As the source is tracked, the peak drifts through in delay space and when it is aligned with the second lag, the second measurement $\boldsymbol{v}_{2}=(0,1,0 \ldots 0)^{\mathrm{T}}$ will be taken (Fig. 14). Repeating this process and gathering these 16 column vectors of measurements gives

$\mathbf{V}_{0}=\left(\begin{array}{llll}\boldsymbol{v}_{1} & \boldsymbol{v}_{2} & \cdots & \boldsymbol{v}_{16}\end{array}\right)$.

In the ideal case, such as this, $\mathbf{V}_{0}$ is the identity matrix $\mathbf{I}$. The matrix $\mathbf{S}_{0}$ is the corresponding collection of ideal spectra from the full calibration process. This means that $\mathbf{S}_{0}$ is equal to the Fourier transform matrix;

$\mathbf{F I}=\mathbf{S}_{0}=\mathbf{F}$.

In reality, there are gain variations, dispersion and non-uniform sampling. For small errors, $\mathbf{V}$ will be close to diagonal. We introduce the recovery matrix $\mathbf{R}$ to find the ideal spectrum $\mathbf{S}_{0}$,

$\mathbf{F R V}=\mathbf{S}_{0}$.

Since $\mathbf{S}_{0} \equiv \mathbf{F}$ from (17),

$\mathbf{R}=\mathbf{V}^{-1}$.

In the real system, the spectrum will not be rectangular. The spectrum for each detector may be recovered by taking the Fourier transform of the detectors' responses over the full calibration run. The individual spectra of the detectors can be accounted for by gathering these measurements into the spectrum matrix $\mathbf{S}$, then the recovery matrix is given by

$\mathbf{R}=\mathbf{F}^{-1} \mathbf{S} \mathbf{V}^{-1}$.
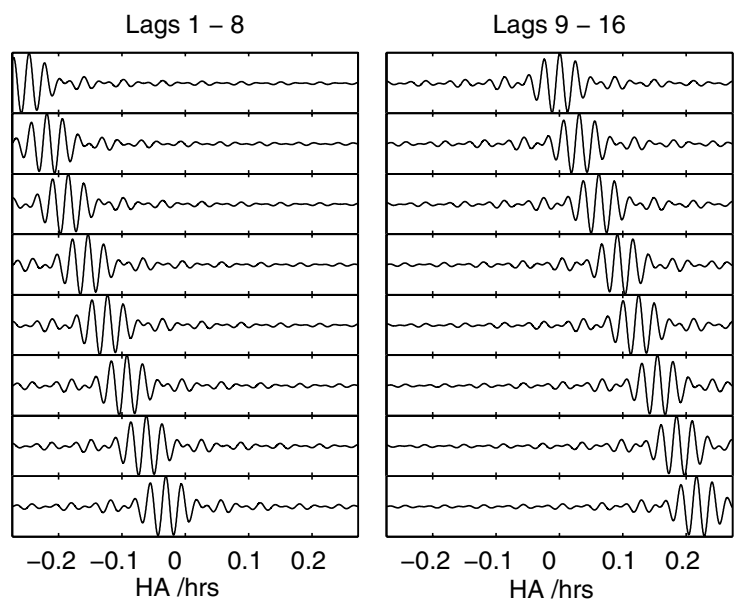

Fig. 14. Lag data during a calibration run on an astronomical source. To calibrate the correlator, a source is tracked with the path compensator held fixed. Unlike earlier plots which were plotted in instrument delay space ( $\tau_{\mathrm{g}}$ constant, $\tau_{\mathrm{i}}$ variable in Eq. (2)), these time-stream data plotted in hour angle which corresponds to $\tau_{\mathrm{i}}$ constant, $\tau_{\mathrm{g}}$ variable, and hence the fringe period is appropriate to the RF frequency $(15 \mathrm{GHz})$ rather than the IF frequency $\left(9 \mathrm{GHz}\right.$ ). The matrix $\mathbf{V}_{0}$ (Eq. (16)) can be constructed from such a data set.

It can be seen from the DFT matrix (Eq. (13)) that the first element of the spectrum vector $s_{0}$ in Eqs. (14) and (15) straddles the zero-frequency band. Because of the choice of lag spacings, this sub-band corresponds to $12 \mathrm{GHz}$ IF. The information content of this zero-frequency band is only half of what it should be because its two halves are redundant. At the other end of the spectrum, the highest frequency sub-band is split between positive and negative frequencies. This causes its centre frequency to be ill-defined. These undesirable symptoms can be avoided by shifting the sub-bands by half channel width using the shift theorem for DFT: multiplying the lag data by a set of complex factors shifts the sub-bands in frequency space. We define a diagonal matrix $\mathbf{H}$ whose diagonal elements are $\left(\epsilon^{0}, \epsilon, \epsilon^{2}, \cdots, \epsilon^{N-1}\right)$, where $\epsilon=\mathrm{e}^{-\pi j / N}$. This channel shift matrix needs to operate before the DFT. The final recovered spectrum is given by

$s_{0}=$ FHR $v$.

Note that $\mathbf{H}$ should not be applied during the calibration step (Eq. (20)) because then $\mathbf{H}$ will be cancelled out.

\subsection{Simulations}

The calibration method was tested on simulated data with analytically-generated astronomical fringes assuming a flat spectrum. The spectrum was evaluated for an ideal correlator with no sampling errors and also with up to $10 \%$ lag errors. Figures $15 \mathrm{a}$ and $\mathrm{b}$ show that the calibrated spectra are similar to the true spectra, while the uncalibrated spectra deviate noticeably. The full spectra, including the negative frequencies, have been shown here. The two halves of the spectrum with channel shift (Fig. 15b) are symmetric and redundant.

The recovered spectra shown in Fig. 15 are not flat, despite the flat band-limited passband that went into modeling the crosscorrelation function. Furthermore, the ripples across the spectrum change as the source drifts through the sky. This is illustrated in Fig. 16. Figure 16a shows a simulation of a processed data stream from a $5 \mathrm{~m}$ east-west baseline with a single point 

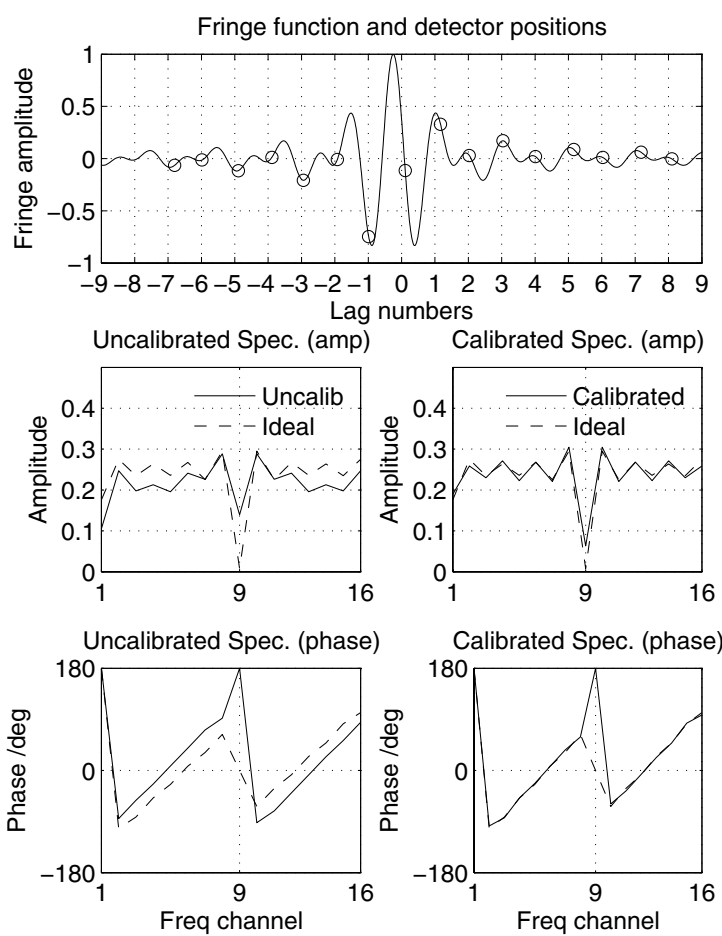

(a) No channel shift
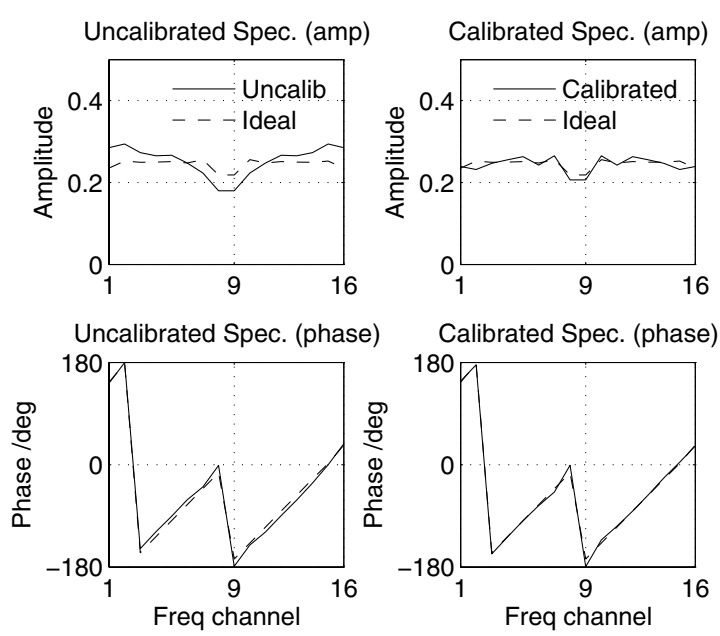

(b) With channel shift

Fig. 15. Calibration can improve the recovered spectrum so that it is closer to what would have been measured by an ideal correlator with no lag errors. Here the lag errors are up to $10 \%$ of the lag spacings. a) The top plot is the response of a point source in instrument delay space. The open circles are the voltages measured at each lag. The lower plots are the amplitudes and phases of the recovered spectra without and with calibration. In b), the channels were shifted. The effect of this is most dramatic in the phase.

source at the centre of the field of view. After fringe-rotation ${ }^{3}$ and standard phase calibration, we expect constant amplitudes

\footnotetext{
${ }^{3}$ In interferometers with independently-mounted antennas, the phase centre drifts as the source is tracked. This changes the phase of each sub-band at a constant rate. The rate is determined by the geometry of the telescope and the frequency of the sub-band. As we know this rate, the varying phase of the sub-bands can be made constant by a process called fringe-rotation. This is equivalent to stopping the fringes in delay space.
}

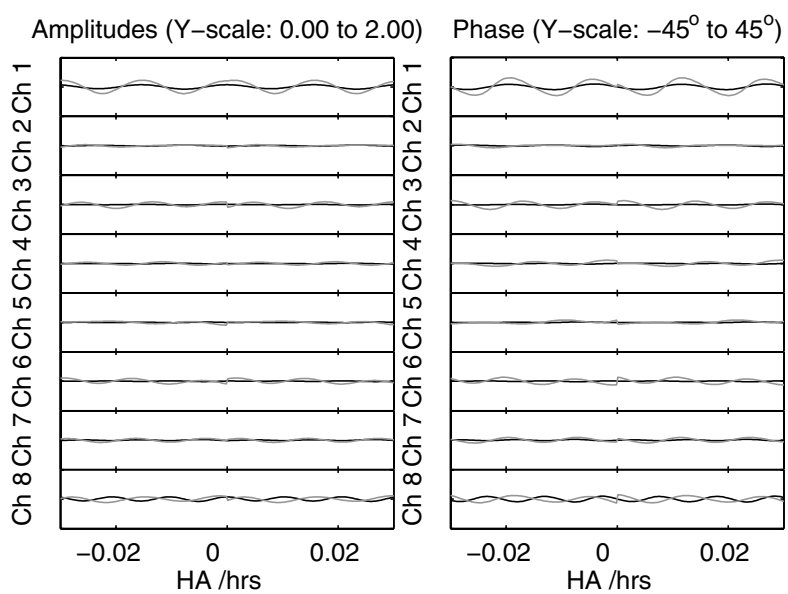

(a) Rectangular window - with and without lag errors
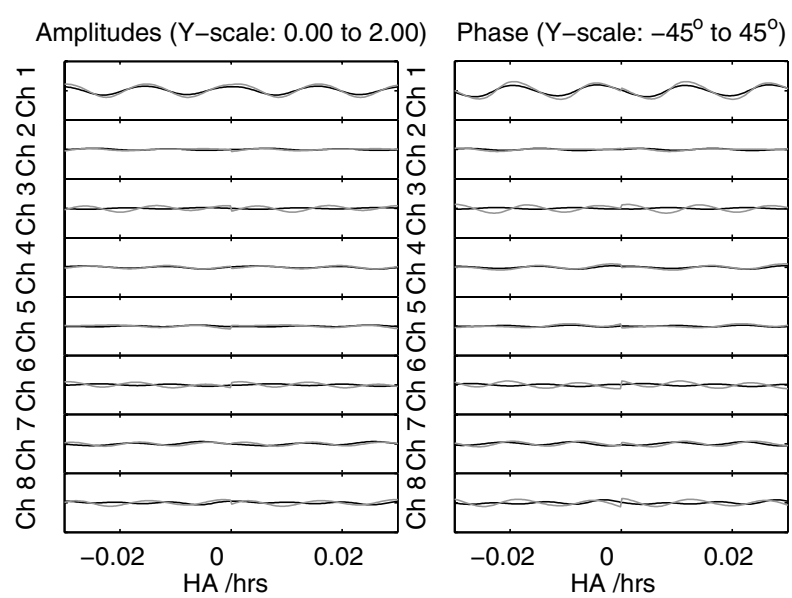

(b) With and without calibration

Fig. 16. Time-stream data of the amplitude and phase for each sub-band. a) compares the time-stream data from an ideal correlator (dark curve) and a correlator with up to 10 percent lag errors (gray curve). It can be seen that lag errors makes alias cycles worse. b) Calibrating the correlator with lag errors makes a small improvement to the magnitude of alias cycles. The gray curves are without calibration and the dark curves are with calibration. The steps in the data are due to the path compensator changing the compensated path.

and zero phase in all sub-bands. Instead we see periodic cycles in both amplitude and phase at the fringe rate for each subband. The magnitude of the cycles are worse for a correlator with lag errors. Calibration does reduce the magnitude of the cycles (Fig. 16b) but it remains noticeably higher than for a correlator with no lag errors (compare Figs. 16a with b).

The cycles arise from aliasing. Because we can only sample the cross-correlation function over some finite range of delays, the recovered spectrum is a convolution of the true spectrum with the spectral response of the window function. But our signal is critically sampled, so the spectrum lies shoulder-to-shoulder with its aliased images (see Fig. 17). The convolved wings of the images extend beyond the $6 \mathrm{GHz}$ bandwidth and alias the signal's spectrum. This is why the edge sub-bands are most affected. Simulations show that narrowing the passband bandwidth by one sub-band reduces these alias cycles, as expected.

Applying a tapered window functions like Hamming window before the DFT makes the alias cycles worse. The response function of the Hamming window has a wider lobe compared 


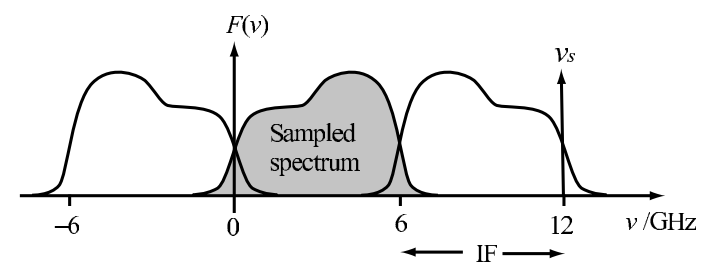

Fig. 17. A 6-12 GHz band-limited signal is sampled at $v_{\mathrm{s}}=12 \mathrm{GHz}$. If we only sample over a finite range of the cross-correlation function, the estimated spectrum is no longer finite and band-limited. Although Nyquist's sampling theorem is apparently satisfied, the recovered spectrum suffers from aliasing.
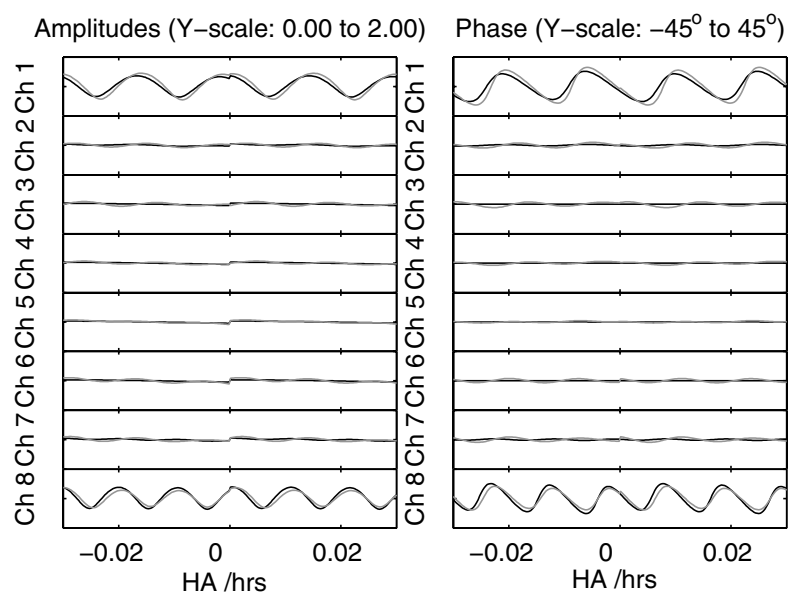

Fig. 18. Time-stream data of the amplitude and phase for each sub-band like Fig. 16a. This time, a Hamming window has been applied and the alias cycles are more severe, particularly in the edge sub-bands. The dark curves are for an ideal correlator and the gray curves are for a correlator with $10 \%$ lag errors.

to a rectangular window (i.e. applying no windowing). So the recovered spectrum will have wider convolved wings and the alias cycles will be worse. However the Hamming window will suppress spectral leakage between channels because it has lower sidelobes $(-43 \mathrm{~dB})$ compared to a rectangular windows (sidelobes up to $-13 \mathrm{~dB}$ ). Applying the Hamming window reduces alias cycles in the central sub-bands but the benefits are marginal (compare Figs. 16a and 18).

Window functions could be beneficial in correlators that oversample the cross-correlation function. For example, if the 6-12 GHz signal is oversampled at $24 \mathrm{GHz}$, half the sub-bands between 0-6 GHz will have no signal. But these empty subbands will act as a buffer to protect the signal from being aliased. A suitable window function with lower sidelobes will suppress aliasing further and reduce spectral leakage between sub-bands. Applying a window function will necessarily broaden the effective spectral resolution. This will need to meet the upper bandwidth limit of the sub-bands (Eq. (3)). It is important to note that not all window functions are appropriate. The Hamming window will taper the sampled cross-correlation function. But it will also down-weight sources at the edge of the field of view because the peak of its correlation function will be offset from the central lag. An offse source's flux density will be systematically underestimated. This bias will vary over the course of an observation (see Fig. 19), so it is impractical to correct for it. We have found that a raised trapezoidal window function works well. It should be flat over the delay range of the field of view and tapered to non-zero levels at the edges (the edge lags contribute some

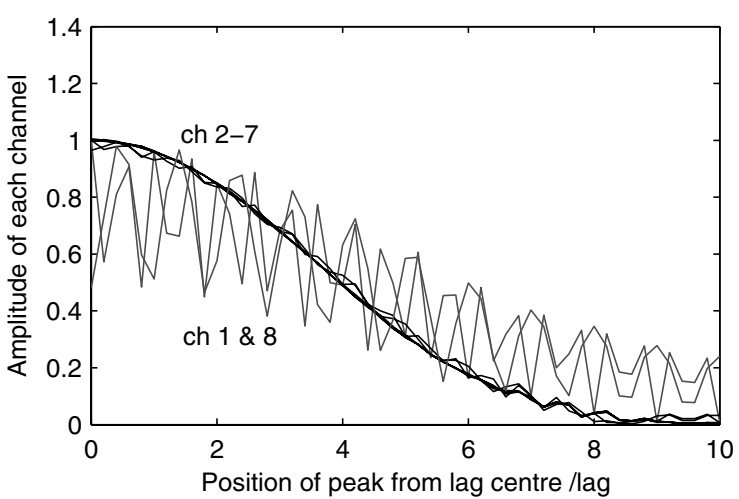

Fig. 19. The amplitude response for each frequency channel for a crosscorrelation function centred away from the centre lag. This could arise from an off-centre source. The plot is the same as Fig. 3 except a Hamming window was used. The window will bias the measured flux density of off-centre sources. While alias cycles in the edge sub-bands (Chaps. 1 and 8) are increased, the alias cycles in the remaining subbands are slightly reduced. The tapered window also has the benefit of suppressing irregular responses in Fig. 3 arising from sources at the edge of the field of view.

useful information). Similarly, a raised version of the more curved Tukey window can work well. A review of window functions can be found in Harris (1978).

The alias cycles are periodic, so the effects are reduced in the final data after smoothing. There will be some loss in the SNR but the introduction of systematic errors is a more serious concern. Residuals will be significant for long fringe periods comparable to the smoothing time. Parts of the data with long fringe periods will need to be edited out. The edge sub-bands suffer most from aliasing. In addition, the noise between sub-bands will be correlated. For these reasons, the edge sub-bands should either be rejected or downweighted.

To summarise, our calibration scheme appears to recover the true spectrum. Simulations suggest that alias cycles will be present in the data as periodic cycles in the estimated spectrum. These are caused by aliasing of the band-limited signal. Applying a window function worsens the effect as does the presence of lag errors in the correlator. Calibration slightly reduced the magnitude of alias cycles. A software-based approach that could overcome aliasing in a narrowband complex correlator will be discussed in a forthcoming paper.

\section{Manufacture and commissioning}

The manufactured version of the correlators were packaged in a $6 \mathrm{U}$ rack-mountable module that can be hot-swapped. The small signal from the detector needs to be buffered immediately, so the detector board was bonded to the amplifier board back-toback. Bonding the PTFE board (with etching on the bottom side) to an FR4 board posed a significant manufacturing issue and could have been simplified had the slot-line phase-shifters been off-board. The whole assembly is housed in an aluminium box for shielding and the lid to the detector board is lined with microwave absorber to prevent standing waves. On the reverse side, a pair of op-amps amplify and remove the DC offset for each detector. The bandwidths of the amplifiers are matched to the video bandwidth of the detectors. 


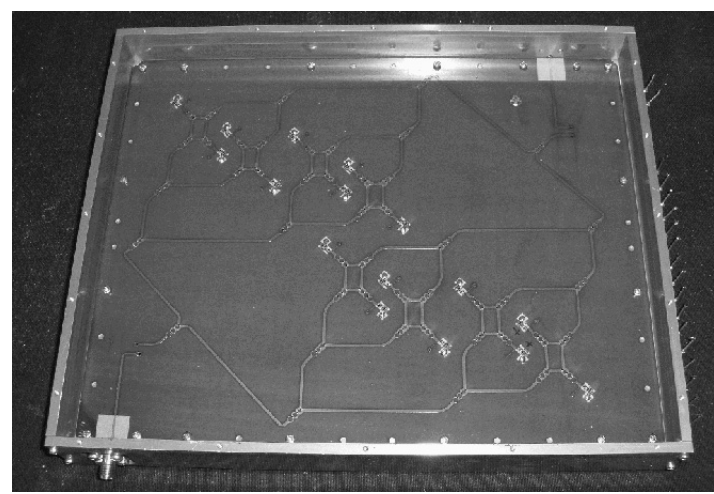

(a) Detector side

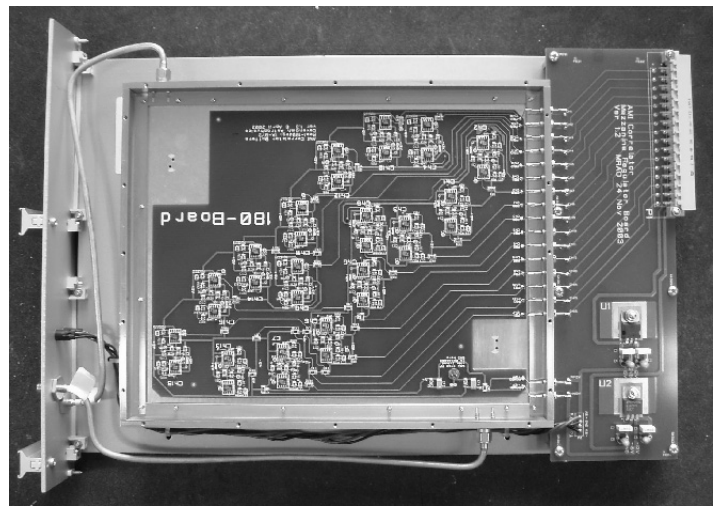

(b) Amplifier side

Fig. 20. The detector side of the correlator board a) and the amplifiers on the other side b). The correlator for each baseline is a self-contained unit.

\subsection{Readout system}

A readout board slots next to the correlator module. It takes the 16 signals from the correlator, demodulates and digitises them. Section 2.3 described how the IF signal at each telescope is phase-switch modulated by orthogonal functions $f$ and $g$ so that the cross-correlated signal can be extracted. The cross-correlated signal can be recovered by demodulating it with $f g$, which can be generated by taking the exclusive-OR of $f$ and $g$. The readout board demodulates the signal by applying a gain of \pm 1 synchronously with the demodulation function.

The number of bits needed by the analogue-to-digital converter (ADC) is determined by the required dynamic range of the telescope. The dynamic range is given by the ratio of the minimum detectable correlated input signal and the correlated signal produced by the strongest source to be observed. At least three bits were assigned to recording the system noise and an additional 11 bits were needed for responding to bright sources ${ }^{4}$. The readout system uses Texas Instrument's 20 bit ADC, DDC112U. The integration time of the readout system was chosen to sample the fastest fringes at least ten times in each fringe cycle. The effective sampling rate is $16 \mathrm{~Hz}$ but the data are stored at a lower rate where possible.

The correlator system needs to be stable over a period of tens of seconds. The $1 / f$ noise of the correlator/readout system has a time constant of around $200 \mathrm{~s}$ with no IF input. When the

${ }^{4}$ We required sufficient dynamic range in the readout system to detect a 100 Jy source without saturating. We assume a telescope with a system temperature of $30 \mathrm{~K}$ and an effective area of $130 \mathrm{~m}^{2}$, together with an integration period of $1 / 32 \mathrm{~s}$.

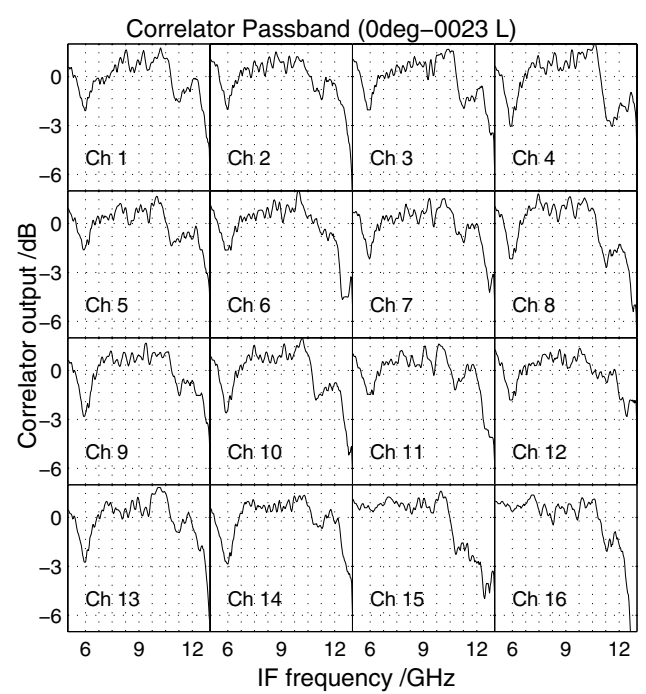

(a) Passbands of the production version correlator

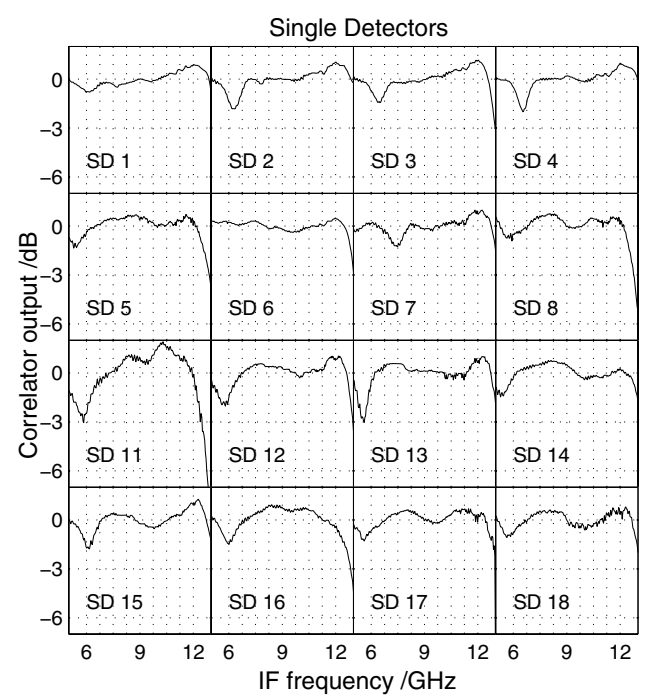

(b) Passbands of the modified single detectors

Fig. 21. a) The IF response of each lag over 5-13 GHz. The boundaries for each of the $0.75 \mathrm{GHz}$ sub-band are shown by dotted lines. Note that unlike the passband for the earlier prototype in Fig. 13a, the passband drops off for the production version drops off above $10.5 \mathrm{GHz}$. b) The IF response of 16 modified detectors.

IF inputs are connected, the $1 / f$ noise is drowned by the telescope's system noise so the correlator system is dominated by the system noise. The performance was compared against the previous generation of readout system developed for the Very Small Array (a CMB interferometer, see Watson et al. 2003). We were able to confirm that their performances were comparable, with the new system achieving slightly better SNR.

\subsection{Passband response}

A problem with the passband of the correlator came to light after it had gone into production. The passband of the correlator was measured by feeding a swept CW signal into one of the ports. The CW was amplitude-modulated by a square wave and the correlator output was demodulated with a similar circuit to the one used in the readout. Figure 21a shows a dip at $6 \mathrm{GHz}$ and 
a fall off above $10.5 \mathrm{GHz}$. Compared with the original design (Fig. 9), we had added a pin to take the signal to the amplifiers on the reverse side. The subsequent reactive load placed on the detector circuit led to the fall-off and coarse structures in the passbands (Fig. 21a).

We manufactured some individual detectors for use as total power detectors to estimate the system temperature. These detectors used the same design as the correlators, except with a resistor in series with the diode to separate the transmission line from the video band circuitry. The passbands of this design are much flatter and do not roll-off below $12 \mathrm{GHz}$ (Fig. 21b). The dip at $6 \mathrm{GHz}$ is still present but at a smaller level. We believe that the $6 \mathrm{GHz}$ dip results from the self-resonant frequency of the lumped components in the detector being comparable to our passband frequency. Smaller bulk components with higher self-resonant frequencies are now available and these may be a solution.

\section{Conclusion}

We have described the design and development of a broad-band (6-12 GHz) analogue lag correlator for radio astronomy applications. Two design approaches were pursued: 1) the real correlator samples the cross-correlation function at Nyquist rate. 2 ) the complex correlator samples both the real and imaginary cross-correlation function but at half-Nyquist rate. Both types were prototyped and tested. The $180^{\circ}$ and $90^{\circ}$ phase shifters used in the designs had errors less than $\pm 6^{\circ}$ and $\pm 15^{\circ}$ respectively over 5-13 GHz. There was very little difference between the two designs in terms of performance. We adopted the real correlator for practical reasons. The passband of the manufactured version rolled off above $10.5 \mathrm{GHz}$ due to a late-stage change to the design. We have shown that with a small modification, the full $6 \mathrm{GHz}$ bandwidth can be achieved. We found unexpected errors in the lag spacings of up to $10 \%$. We note that dispersion in the microstrip delay lines $\left( \pm 25^{\circ}\right.$ across the band) may cause problems, particularly for the outer lags. However, the effects are negligible in comparison to the lag errors. We have described a practical calibration scheme that uses an astronomical source to overcome delay errors and gain variations in the lags, as well as dispersion. Simulations show that the edge channels will be aliased in a critically-sampled correlator like ours. We will explore this issue in a forthcoming paper.

Acknowledgements. The authors would like to thank Chris Clementson, Will Grainger, Angela Taylor, Dave Titterington, Vic Quy and the other members of the AMI collaboration for their contributions to the development and testing of the correlator system.

\section{References}

Bagchi, S., \& Mitra, S. K. 1998, Nonuniform Discrete Fourier Transform and Its Signal Processing Applications (Norwell MA: Kluwer Academic Publishers) Bracewell, R. 1999, Fourier Transform and Its Applications (McGraw Hill Higher Education)

Harris, A. I. 2003, in Proc. of the SPIE; Millimeter and Submillimeter Detectors for Astronomy, ed. T. G. Phillips, \& J. Zmuidzinas, 279

Harris, A. I., \& Zmuidzinas, J. 2001, Rev. Scient. Instr., 72, 1531

Harris, F. J. 1978, Proc. of the IEEE, 66, 51

Hewlett-Packard. AN 986, Square Law and Linear Detection, Application Note 986

Holler, C. M., \& Jones, M. E. 2007, IEEE Microwave and Wireless Components Letters (submitted)

Jones, M. E. 2002, in AMiBA 2001: High-Z Clusters, Missing Baryons, and CMB Polarization, ed. L.-W. Chen, C.-P. Ma, K.-W. Ng, \& U.-L. Pen, ASP Conf. Ser., 257, 35

Kaneko, T., \& the AMI Collaboration. 2006, in Proc. of SPIE; Ground-based and Airborne Telescopes, ed. L. M. Stepp, Vol. 6267

Kneissl, R., Jones, M. E., Saunders, R., et al. 2001, MNRAS, 328, 783

Leitch, E. M., Pryke, C., Halverson, N. W., et al. 2002, ApJ, 568, 28

Li, C.-T., Kubo, D., Han, C.-C., et al. 2004, in Proc. of the SPIE, ed. C. M. Bradford, P. A. R. Ade, J. E. Aguirre, et al., 5498, 455

Lo, K., Martin, R., \& Chiueh, T. 2001, in 2001 Asia-Pacific Radio Science Conference AP-RASC '01, 235

O'Sullivan, C., Yassin, G., Woan, G., et al. 1995, MNRAS, 274, 861

Padin, S., Cartwright, J. K., Shepherd, M. C., Yamasaki, J. K., \& Holzapfel, W. L. 2001, IEEE Trans. Instrum. Meas., 50, 1234

Padin, S., Shepherd, M. C., Cartwright, J. K., et al. 2002, PASP, 114, 83

Roberts, P. P., Leach, M. R., \& Wilson, W. E. 2007, PASP (submitted)

Ryle, M. 1952, Roy. Soc. London Proc. Ser. A, 211, 351

Sunyaev, R. A., \& Zel'dovich, Y. B. 1972, Commun. Astrophys. Space Phys., 4

Thompson, A. R., Moran, J. M., \& Swenson, G. W. 2001, Interferometry and Synthesis in Radio Astronomy, 2nd edn. (John Wiley \& Sons)

Watson, R. A., Carreira, P., Cleary, K., et al. 2003, MNRAS, 341, 1057 